\title{
INVERSION OF L-FUNCTIONS, GENERAL KLOOSTERMAN SUMS WEIGHTED BY INCOMPLETE CHARACTER SUMS
}

\author{
Xiaobeng Zhang and Huaning LiU
}

\begin{abstract}
The main purpose of this paper is using estimates for character sums and analytic methods to study the mean value involving the incomplete character sums, 2-th power mean of the inversion of Dirichlet $L$-function and general Kloosterman sums, and give four interesting asymptotic formulae for it.
\end{abstract}

\section{$\S 1$. Introduction}

Let $q \geq 3$ be an integer, $\chi$ be a Dirichlet character modulo $q$, and $L(s, \chi)$ be the Dirichlet $L$-function corresponding to $\chi$. The estimate for character sums is of great importance in number theory. The various arithmetical properties of $\sum_{n=N+1}^{N+H} \chi(n)$ were investigated by many authors. For example, the classical result, due to Pólya and Vinogradov (see Theorem 8.21 of [1]), is that the estimates

$$
\sum_{n=N+1}^{N+H} \chi(n) \ll q^{\frac{1}{2}} \ln q
$$

hold for all non-principal character $\chi$ modulo $q$. To higher moments, H. L. Montgomery and R. C. Vaughan (see [4]) established a non-trivial bound and proved that for any positive integer $k$,

$$
\sum_{\substack{\chi \bmod q \\ \chi \neq \chi_{0}}} \max _{1 \leq y \leq q}\left|\sum_{n \leq y} \chi(n)\right|^{2 k} \ll \phi(q) q^{2 k} .
$$

Received December 12, 2008; Revised January 4, 2009.

2000 Mathematics Subject Classification. 11L05.

Key words and phrases. character sum, $L$-function, general Kloosterman sum, asymptotic formula.

This work is supported by the $\operatorname{NSF}(10671155)$ of P. R. China and the Natural Science Foundation of the Education Department of Shaanxi Province of China under Grant (08JK437). 
For applications to arithmetic, one also needs non-trivial bounds for incomplete character sums. For that purpose, Z. Xu and W. Zhang (see [6]) transformed the character sums over quarter intervals to $L$-functions and studied the $2 k$-th power mean value of the incomplete character sums, and provided a sharper asymptotic formula as follows:

$$
\begin{aligned}
& \sum_{\substack{\chi \bmod q \\
\chi(-1)=1}}^{*}\left|\sum_{x<\frac{q}{4}} \chi(x)\right|^{2 k} \\
= & \frac{J(q) q^{k}}{16}\left(\frac{\pi}{8}\right)^{2 k-2} \prod_{p \mid q}\left(1-\frac{1}{p^{2}}\right)^{2 k-1} \prod_{p \nmid 2 q}\left(1-\frac{1-C_{2 k-2}^{k-1}}{p^{2}}\right)+O\left(q^{k+\epsilon}\right),
\end{aligned}
$$

where $\sum^{*} \chi(-1)=1$ denotes the summation over all primitive characters modulo $q$ such that $\chi(-1)=1, J(q)$ denotes the number of all primitive characters modulo $q, \prod_{p \mid q}$ denotes the product over all prime divisors $p$ of $q, C_{n}^{m}=$ $\frac{n !}{m !(n-m) !}$ and $\epsilon$ is any fixed positive number.

Concerning the general non-principal character modulo $q$, W. Zhang (see [7]) studied the mean value of $L$-functions with the weight of incomplete character sums and obtained that for any real number $N$ with $1<N<\sqrt{q}$,

$$
\begin{aligned}
& \sum_{\substack{\chi \bmod q \\
\chi \neq \chi_{0}}}\left|\sum_{n \leq N} \chi(n)\right|^{2} \cdot|L(1, \chi)|^{2} \\
= & \left(\frac{\pi^{2}}{6}\right)\left(\frac{\phi^{2}(q)}{q}\right) N \prod_{p \mid q}\left(1-\frac{1}{p^{2}}\right)+O\left(\phi(q) 2^{\omega(q)} \ln ^{2} q\right) \\
+ & \left(\frac{\pi^{2}}{3}\right)\left(\frac{\phi^{2}(q)}{q}\right) \frac{N}{\zeta(3)} \prod_{p \mid q}\left(1-\frac{1}{p^{2}+p+1}\right) \\
& \times \sum_{\substack{m=1 \\
(m, q)=1 \\
(m+n, q)=1}}^{\infty} \sum_{\substack{n=1 \\
(n, q)=1}}^{\infty} \frac{1}{m n(m+n)}+O\left(N^{3} \ln ^{2} q\right),
\end{aligned}
$$

where $\sum_{\chi \neq \chi_{0}}$ denotes the summation over all non-principal characters modulo $q, \phi(q)$ is the Euler function and $\omega(q)$ denotes the number of all different prime divisors of $q$.

For any integers $r$ and $s$, we define the general Kloosterman sums $K(r, s, \chi ; q)$ as follows:

$$
K(r, s, \chi ; q)=\sum_{\substack{a=1 \\(a, q)=1}}^{q} \chi(a) e\left(\frac{r a+s \bar{a}}{q}\right)
$$


where $a \bar{a} \equiv 1 \bmod q ; \chi$ denotes a Dirichlet character $\bmod q$ and $e(y)=e^{2 \pi i y}$. This summation is very important, because it is a generalization of the classical Kloosterman sums $K\left(r, s, \chi_{0} ; q\right)=K(r, s, q)$, where $\chi_{0}$ is the principal character $\bmod q$. Perhaps the most famous properties of $K(r, s, \chi ; q)$ is the estimate (see $[2])$ :

$$
|K(r, s, \chi ; p)| \ll(r, s, p)^{\frac{1}{2}} p^{\frac{1}{2}+\epsilon},
$$

where $p$ is a prime, $(r, s, p)$ denotes the great common divisor of $r, s$ and $p$, $\epsilon$ is any fixed positive number. For an arbitrary composite number $q$, we do not know how large $|K(r, s, \chi, q)|$ is. In fact, the value of $|K(r, s, \chi, q)|$ is quite irregular when $q$ is not a prime.

However, it is surprising that $|K(r, s, \chi, q)|$ enjoys many good values distribution properties. W. Zhang proved the following (see [8]):

$$
\begin{aligned}
& \sum_{\chi \bmod } \sum_{q}^{q}|K(r, s, \chi, q)|^{4} \\
= & \phi^{2}(q) q^{2} d(q) \prod_{p^{\alpha} \| q}\left(1-\frac{2}{\alpha+1} \frac{p^{\alpha-1}-1}{p^{\alpha}(p-1)}+\frac{\alpha-4 p^{\alpha-1}}{(\alpha+1) p^{\alpha}}\right) .
\end{aligned}
$$

Furthermore, he also studied the mean value of Dirichlet $L$-functions with the weight of general Kloosterman sums, and obtained the following interesting asymptotic formula (see [9]):

$$
\begin{aligned}
& \sum_{\substack{\chi \bmod _{\chi \neq} \\
\chi \neq \chi_{0}}}|K(r, s, \chi, q)|^{2} \cdot|L(1, \chi)|^{2 k} \\
= & \left(\frac{\pi^{2}}{6}\right)^{2 k-1} \phi^{2}(q) \prod_{p \mid q}\left(1-\frac{1}{p^{2}}\right)^{2 k-1} \prod_{p \nmid q}\left(1-\frac{1-C_{2 k-2}^{k-1}}{p^{2}}\right)+O\left(q^{\frac{3}{2}+\epsilon}\right),
\end{aligned}
$$

where $(r s, q)=1$.

It is interesting to consider more mean value of character sums. Due to the results of [7], a natural question arises: What happens if we impose on the inversion of Dirichlet $L$-function and general Kloosterman sums to be weighted by incomplete character sums. In this paper, we shall use the estimates for character sums and analytic methods to study the mean value of 2 -th power mean of the inversion of Dirichlet $L$-function, general Kloosterman sums weighted by incomplete character sums, four interesting asymptotic formulae are obtained extending the results of [7].

That is, we shall prove the following:

Theorem 1. Let $q \geq 3$ be an integer. Then on the assumption of generalized Riemann hypothesis for Dirichlet $L$-function $L(s, \chi)$ and for any real number 
$N$ with $1<N<\sqrt{q}$, we have the asymptotic formula

$$
\begin{aligned}
& \sum_{\substack{\chi \bmod _{q} \neq \chi_{0} \\
\chi \neq N}}\left|\sum_{n \leq N} \chi(n)\right|^{2} \cdot \frac{1}{|L(1, \chi)|^{2}} \\
= & \frac{\zeta(2)}{\zeta(4)} \frac{\phi^{2}(q)}{q} N \prod_{p \mid q}\left(1-\frac{1}{p^{2}+1}\right)(1+C)+O\left(\phi(q) 2^{\omega(q)}\right)+O\left(N^{3} \ln ^{2} q\right),
\end{aligned}
$$

where $\sum_{\chi \neq \chi_{0}}$ denotes the summation over all non-principal characters modulo $q, \zeta(s)$ is the Riemann zeta-function, $\phi(q)$ is the Euler function, $\prod_{p \mid q}$ denotes the product over all different prime divisors of $q, \omega(q)$ denotes the number of all different prime divisors of $q$,

$$
C=2 \sum_{\substack{n_{1}=1 \\\left(m_{1}, n_{1}\right)=1}}^{\infty} \sum_{\substack{m_{1}=n_{1}+1 \\\left(t, m_{1}\right)=1}}^{\infty} \sum_{\substack{t \mid m_{1} \\ n_{1}}}^{\prime} \frac{\mu\left(n_{1}\right) \mu\left(m_{1}\right) \mu(t) \mu(e)}{n_{1} m^{2} t^{2} e^{2}}
$$

is a constant depending only on $\bmod q, \sum_{m=1}^{\prime \infty}$ denotes the summation over all $m$ such that $(m, q)=1$ and $\mu(n)$ is the Möbius function.

Theorem 2. Let $q \geq 3$ be an integer. Then for any real number $N$ with $1<N<\sqrt{q}$ and integers $r$ and $s$ with $(r s, q)=1$, we have the asymptotic formula

$$
\sum_{\substack{\chi \bmod q \\ \chi \neq \chi_{0}}}\left|\sum_{n \leq N} \chi(n)\right|^{2} \cdot|K(r, s, \chi ; q)|^{2}=\frac{\phi^{3}(q) N}{q}+O\left(N^{2} q^{\frac{3}{2}} d^{2}(q)\right) .
$$

Theorem 3. Let $q \geq 3$ be an integer. Then for any real number $N$ with $1<N<q^{\frac{1}{4}}$ and integers $r$ and $s$ with $(r s, q)=1$, we have the asymptotic formula

$$
\begin{aligned}
& \sum_{\substack{\chi \bmod q \\
\chi \neq \chi_{0}}}\left|\sum_{n \leq N} \chi(n)\right|^{4} \cdot|K(r, s, \chi ; q)|^{2} \\
= & \frac{12 \phi^{6}(q) N^{2}}{\pi^{2} q^{4}} \prod_{p \mid q}\left(1-\frac{1}{p^{2}}\right)^{-1} \ln N+O\left(\phi^{4}(q) q^{-2} N^{2} d(q)\right) .
\end{aligned}
$$

Theorem 4. Let $p>2$ be a prime. Then for any real number $N$ with $1<$ $N<p^{\frac{1}{4}}$ and integers $r$ and $s$ with $(r s, p)=1$, we have the asymptotic formula

$$
\sum_{\substack{\chi \bmod p \\ \chi \neq \chi_{0}}}\left|\sum_{n \leq N} \chi(n)\right|^{4} \cdot|K(r, s, \chi ; p)|^{2}
$$




$$
=p^{2} N^{2}\left(\frac{12(\ln N+\gamma)}{\pi^{2}}-\frac{72 \zeta^{\prime}(2)+\pi^{4}}{\pi^{4}}\right)+O\left(p^{2} N \ln N\right)+O\left(N^{4} p^{\frac{3}{2}}\right),
$$

where $\gamma$ is the Euler constant and $\zeta(s)$ is the Riemann zeta-function.

We believe that our method can be applied to sums of $2 k$-th $(k \geq 3)$ power mean

$$
\sum_{\substack{\chi \bmod q \\ \chi \neq \chi_{0}}}\left|\sum_{n \leq N} \chi(n)\right|^{2 k} \cdot|K(r, s, \chi ; q)|^{2}
$$

but the constant will be very complicate. So we do not give a general conclusion here.

Unfortunately we still do not know how to estimate more general sums

$$
\sum_{\substack{\chi \bmod q \\ \chi \neq \chi_{0}}}\left|\sum_{n \leq N} \chi(n)\right|^{2} \cdot \frac{1}{|L(1, \chi)|^{2 k}} \text {. }
$$

For general integer $k \geq 3$, whether there exist an asymptotic formula for (1.1) is also an open problem.

\section{$\S 2$. Several lemmas}

In order to complete the proof of the theorems, we need several lemmas.

Lemma 1. Let $q>2$ be a positive integer, a be any integer with $1 \leq a \leq q$ and $(a, q)=1$. Then on the assumption of generalized Riemann hypothesis for Dirichlet $L$-function $L(s, \chi)$ we have the asymptotic formula

$$
\sum_{\substack{\chi \bmod q \\ \chi \neq \chi_{0}}} \chi(a) \cdot \frac{1}{|L(1, \chi)|^{2}}=\sum_{\substack{\chi \bmod q \\ \chi \neq \chi_{0}}} \chi(a) \cdot\left|\sum_{1 \leq n \leq q^{4}} \frac{\chi(n) \mu(n)}{n}\right|^{2}+O\left(q^{-1+\epsilon}\right),
$$

where $\mu(n)$ is the Möbius function and $\epsilon$ is any fixed positive number.

Proof. Firstly we let

$$
A(\chi, y)=\sum_{q^{4}<n \leq y} \chi(n) \mu(n) .
$$

On the assumption of generalized Riemann hypothesis for Dirichlet $L$-function $L(s, \chi)$ we know

$$
A(\chi, y)=\sum_{q^{4}<n \leq y} \chi(n) \mu(n) \ll y^{\frac{1}{2}+\epsilon},
$$

where $\epsilon$ is any fixed positive number. If $\operatorname{Re}(s)>1$ and $\chi \neq \chi_{0}$, then we have

$$
\frac{1}{L(s, \chi)}=\sum_{n=1}^{\infty} \frac{\chi(n) \mu(n)}{n^{s}}
$$


From the above and the Abel's summation we may get

$$
\frac{1}{L(s, \chi)}=\sum_{1 \leq n \leq q^{4}} \frac{\chi(n) \mu(n)}{n^{s}}+s \int_{q^{4}}^{+\infty} \frac{A(\chi, y)}{y^{s+1}} d y
$$

By the properties of Dirichlet $L$-functions and $L(1, \chi) \neq 0$, we know that $(2.1)$ is also correct if $s=1$. So we have

$$
\begin{aligned}
& \sum_{\substack{\chi \bmod q \\
\chi \neq \chi_{0}}} \chi(a) \cdot \frac{1}{|L(1, \chi)|^{2}} \\
& =\sum_{\substack{\chi \bmod q \\
\chi \neq \chi_{0}}} \chi(a)\left|\sum_{1 \leq n \leq q^{4}} \frac{\chi(n) \mu(n)}{n}+\int_{q^{4}}^{+\infty} \frac{A(\chi, y)}{y^{2}} d y\right|^{2} \\
& =\sum_{\substack{\chi \bmod q \\
\chi \neq \chi_{0}}} \chi(a)\left|\sum_{1 \leq n \leq q^{4}} \frac{\chi(n) \mu(n)}{n}\right|^{2} \\
& +\sum_{\substack{\chi \bmod q \\
\chi \neq \chi_{0}}} \chi(a)\left(\sum_{1 \leq n \leq q^{4}} \frac{\chi(n) \mu(n)}{n}\right)\left(\int_{q^{4}}^{+\infty} \frac{A(\bar{\chi}, y)}{y^{2}} d y\right) \\
& +\sum_{\substack{\chi \bmod q \\
\chi \neq \chi_{0}}} \chi(a)\left(\sum_{1 \leq n \leq q^{4}} \frac{\bar{\chi}(n) \mu(n)}{n}\right)\left(\int_{q^{4}}^{+\infty} \frac{A(\chi, y)}{y^{2}} d y\right) \\
& +\sum_{\substack{\chi \bmod q \\
\chi \neq \chi_{0}}} \chi(a)\left|\int_{q^{4}}^{+\infty} \frac{A(\chi, y)}{y^{2}} d y\right|^{2} \text {. }
\end{aligned}
$$

Using the Cauchy inequality, we have the estimates (2.3)

$$
\begin{aligned}
& \left|\sum_{\substack{\chi \bmod q \\
\chi \neq \chi_{0}}} \chi(a)\left(\sum_{1 \leq n \leq q^{4}} \frac{\chi(n) \mu(n)}{n}\right)\left(\int_{q^{4}}^{+\infty} \frac{A(\bar{\chi}, y)}{y^{2}} d y\right)\right| \\
& \ll\left[\sum_{\substack{\chi \bmod q \\
\chi \neq \chi_{0}}}\left|\sum_{1 \leq n \leq q^{4}} \frac{\chi(n) \mu(n)}{n}\right|^{2}\right]^{\frac{1}{2}}\left[\sum_{\substack{\bmod _{1} \\
\chi \neq \chi_{0}}}\left|\int_{q^{4}}^{+\infty} \frac{A(\chi, y)}{y^{2}} d y\right|^{2}\right]^{\frac{1}{2}} \ll q^{-1+\epsilon},
\end{aligned}
$$




$$
\left|\sum_{\substack{\chi \bmod q \\ \chi \neq \chi_{0}}} \chi(a)\left(\sum_{1 \leq n \leq q^{4}} \frac{\bar{\chi}(n) \mu(n)}{n}\right)\left(\int_{q^{4}}^{+\infty} \frac{A(\chi, y)}{y^{2}} d y\right)\right| \ll q^{-1+\epsilon}
$$

and

$$
\left.\left|\sum_{\substack{\chi \bmod q \\ \chi \neq \chi_{0}}} \chi(a)\right| \int_{q^{4}}^{+\infty} \frac{A(\chi, y)}{y^{2}} d y\right|^{2} \mid \ll q^{-3+\epsilon} .
$$

Combining (2.2)-(2.4) and (2.5) we may immediately get the asymptotic formula

$$
\sum_{\substack{\chi \bmod q \\ \chi \neq \chi_{0}}} \chi(a) \cdot \frac{1}{|L(1, \chi)|^{2}}=\sum_{\substack{\chi \bmod q \\ \chi \neq \chi_{0}}} \chi(a) \cdot\left|\sum_{1 \leq n \leq q^{4}} \frac{\chi(n) \mu(n)}{n}\right|^{2}+O\left(q^{-1+\epsilon}\right) .
$$

This proves Lemma 1.

Lemma 2. Let $q>2$ be a positive integer. Then for any positive integers $m$ and $n$ and any real number $N>1$ we have the asymptotic formula

$$
\sum_{\substack{1<m \leq N \\(m, n)=1}}^{\prime} \sum_{1 \leq n<m}^{\prime} \frac{1}{m^{2}}=\frac{6 \phi^{2}(q)}{\pi^{2} q^{2}} \prod_{p \mid q}\left(1-\frac{1}{p^{2}}\right)^{-1} \ln N+O(d(q)),
$$

where $\sum_{m \leq N}^{\prime}$ denotes the summation over all $1 \leq m \leq N$ with $(m, q)=1$, $\phi(q)$ is the Euler function and $\prod_{p \mid q}$ denotes the product over all different prime divisors of $q$.

Proof. Note that

$$
\sum_{\substack{m \leq N \\(m, q)=1}} 1=\frac{\phi(q)}{q} N+O\left(2^{\omega(q)}\right)
$$

and

$$
\frac{\phi(m)}{m}=\sum_{d \mid m} \frac{\mu(d)}{d},
$$

where $\omega(q)$ denotes the number of all different prime divisors of $q$.

We have

$$
\sum_{\substack{1<m \leq N \\(m, n)=1}}^{\prime} \sum_{1 \leq n<m}^{\prime} \frac{1}{m^{2}}=\sum_{\substack{1<m \leq N \\(m, n)=1 \\(m, q)=1}} \sum_{\substack{1 \leq n<m \\(m)}} \frac{1}{m^{2}} \sum_{\substack{d|n \\ d| q}} \mu(d)
$$




$$
\begin{aligned}
& =\sum_{\substack{1<m \leq N \\
(m, q)=1}} \frac{1}{m^{2}} \sum_{\substack{d \mid q \\
(d, m)=1}} \mu(d) \sum_{\substack{1 \leq k<m / d \\
(k, m)=1}} 1 \\
& =\sum_{\substack{1<m \leq N \\
(m, q)=1}} \frac{1}{m^{2}} \sum_{\substack{d \mid q \\
(d, m)=1}} \mu(d)\left(\frac{m}{d} \frac{\phi(m)}{m}+O\left(2^{\omega(m)}\right)\right) \\
& =\sum_{\substack{1<m \leq N \\
(m, q)=1}} \frac{\phi(m)}{m^{2}} \sum_{d \mid q} \frac{\mu(d)}{d}+O(d(q)) \\
& =\frac{\phi(q)}{q}\left(\sum_{\substack{1 \leq m \leq N \\
(m, q)=1}} \frac{\phi(m)}{m^{2}}-1\right)+O(d(q)) .
\end{aligned}
$$

We also have the asymptotic formulae

$$
\begin{gathered}
\sum_{\substack{m \leq N \\
(m, q)=1}} \frac{1}{m}=\frac{\phi(q)}{q}\left(\ln N+\gamma+\sum_{p \mid q} \frac{\ln p}{p-1}\right)+O\left(\frac{2^{\omega(q)}}{N}\right) ; \\
\sum_{\substack{d \leq N \\
(d, q)=1}} \frac{\mu(d)}{d^{2}}=\frac{6}{\pi^{2}} \prod_{p \mid q}\left(1-\frac{1}{p^{2}}\right)^{-1}+O\left(\frac{1}{N}\right)
\end{gathered}
$$

and

$$
\sum_{\substack{d \leq N \\(d, q)=1}} \frac{\mu(d) \ln d}{d^{2}}=\sum_{\substack{d=1 \\(d, q)=1}}^{\infty} \frac{\mu(d)}{d^{2}}\left(-\sum_{m=1}^{\infty} \frac{\Lambda(m)}{m^{2}}+\sum_{p \mid q} \frac{\ln p}{p^{2}-1}\right)+O\left(\frac{\ln N}{N}\right)
$$

where $\gamma$ is the Euler constant, $\Lambda(m)$ is the Mangoldt function, $\prod_{p \mid q}$ denotes the product over all different prime divisors of $q$ and $\sum_{p \mid q}$ denotes the summation over all different prime divisors of $q$.

Combing (2.7), (2.8), and (2.9) we can get

$$
\begin{aligned}
& \sum_{\substack{m=1 \\
(m, q)=1}}^{N} \frac{\phi(m)}{m^{2}}=\sum_{\substack{1 \leq l d \leq N \\
(l d, q)=1}} \frac{\mu(d)}{d^{2} \cdot l}=\sum_{\substack{1 \leq d \leq N \\
(d, q)=1}} \frac{\mu(d)}{d^{2}} \sum_{\substack{1 \leq l \leq N / d \\
(l, q)=1}} \frac{1}{l} \\
= & \sum_{\substack{1 \leq d \leq N \\
(d, q)=1}} \frac{\mu(d)}{d^{2}}\left[\frac{\phi(q)}{q}\left(\ln \frac{N}{d}+\gamma+\sum_{p \mid q} \frac{\ln p}{p-1}\right)+O\left(\frac{d \cdot 2^{\omega(q)}}{N}\right)\right]
\end{aligned}
$$




$$
\begin{aligned}
= & \frac{\phi(q)}{q}\left(\ln N+\gamma+\sum_{p \mid q} \frac{\ln p}{p-1}\right) \sum_{\substack{1 \leq d \leq N \\
(d, q)=1}} \frac{\mu(d)}{d^{2}}-\frac{\phi(q)}{q} \sum_{\substack{1 \leq d \leq N \\
(d, q)=1}} \frac{\mu(d)}{d^{2}} \ln d \\
& +O\left(\sum_{1 \leq d \leq N} \frac{1}{d} \frac{2^{\omega(q)}}{N}\right) \\
= & \frac{6 \phi(q)}{\pi^{2} q} \prod_{p \mid q}\left(1-\frac{1}{p^{2}}\right)^{-1}\left(\ln N+\gamma+\sum_{m=1}^{\infty} \frac{\Lambda(m)}{m^{2}}+\sum_{p \mid q} \frac{p \cdot \ln p}{p^{2}-1}\right) \\
& +O\left(\frac{2^{\omega(q)} \ln N}{N}\right) .
\end{aligned}
$$

From (2.6) and (2.10) we immediately proves Lemma 2.

Lemma 3. Let $p>2$ be a prime. Then for any positive integers $m$ and $n$ and for any real number $N$ with $1<N<p$ we have the asymptotic formula

$$
\sum_{\substack{1<m \leq N \\(m, n)=1}}^{\prime} \sum_{1 \leq n<m}^{\prime} \frac{1}{m^{2}}=(\ln N+\gamma) \frac{6}{\pi^{2}}-\frac{36}{\pi^{4}} \zeta^{\prime}(2)-1+O\left(\frac{\ln N}{N}\right),
$$

where $\sum_{m \leq N}^{\prime}$ denotes the summation over all $1 \leq m \leq N$ with $(m, p)=1, \gamma$ is the Euler constant and $\zeta(s)$ is the Riemann zeta-function.

Proof. Since the number of positive integers $n \leq m$ that are relatively prime is $\phi(m)$, so we have

$$
\sum_{\substack{1<m \leq N \\(m, n)=1}}^{\prime} \sum_{\substack{1 \leq n<m \\(m)}}^{\prime} \frac{1}{m^{2}}=\sum_{m=2}^{N} \frac{\phi(m)}{m^{2}}=\sum_{m=1}^{N} \frac{\phi(m)}{m^{2}}-1,
$$

where $\phi(m)$ denotes the Euler function. Note that

$$
\sum_{n=1}^{\infty} \frac{\mu(n)}{n^{2}} \ln n=\frac{\zeta^{\prime}(2)}{\zeta^{2}(2)}=\frac{36}{\pi^{4}} \zeta^{\prime}(2)
$$

and

$$
\sum_{n<x} \frac{1}{n}=\ln x+\gamma+O\left(\frac{1}{x}\right)
$$

where $\zeta(s)$ is the Riemann zeta-function and $\gamma$ is the Euler constant. We can get

$$
\sum_{m=1}^{N} \frac{\phi(m)}{m^{2}}=\sum_{m=1}^{N} \frac{1}{m} \sum_{d \mid m} \frac{\mu(d)}{d}=\sum_{d \leq N} \frac{\mu(d)}{d^{2}} \sum_{h \leq N / d} \frac{1}{h}
$$




$$
\begin{aligned}
& =\sum_{d \leq N} \frac{\mu(d)}{d^{2}}\left(\ln \frac{N}{d}+\gamma+O\left(\frac{d}{N}\right)\right) \\
& =(\ln N+\gamma) \sum_{d \leq N} \frac{\mu(d)}{d^{2}}-\sum_{d \leq N} \frac{\mu(d)}{d^{2}} \ln d+O\left(\frac{1}{N} \sum_{d \leq N} \frac{1}{d}\right) \\
& =(\ln N+\gamma) \frac{6}{\pi^{2}}+O\left(\ln N \sum_{m>N} \frac{1}{m^{2}}\right)-\frac{36}{\pi^{4}} \zeta^{\prime}(2)+O\left(\sum_{d>N} \frac{\ln d}{d^{2}}\right)+O\left(\frac{\ln N}{N}\right) \\
& =(\ln N+\gamma) \frac{6}{\pi^{2}}-\frac{36}{\pi^{4}} \zeta^{\prime}(2)+O\left(\frac{\ln N}{N}\right) .
\end{aligned}
$$

Using (2.11) and above estimates we immediately proves Lemma 3.

\section{$\S 3$. Proof of the theorems}

In this section, we shall complete the proof of the theorems. First using Lemma 1 and the methods of [7] we have

$$
\begin{aligned}
& \sum_{\substack{\chi \bmod q \\
\chi \neq \chi_{0}}}\left|\sum_{n \leq N} \chi(n)\right|^{2} \cdot \frac{1}{|L(1, \chi)|^{2}} \\
& =\sum_{\chi \bmod } \sum_{q \leq N}^{\prime} \sum_{n \leq N}^{\prime} \chi(n \bar{m}) \frac{1}{|L(1, \chi)|^{2}} \\
& =\sum_{m \leq N}^{\prime} \sum_{n \leq N}^{\prime} \sum_{\substack{\bmod q \\
\chi \neq \chi_{0}}} \chi(n \bar{m}) \frac{1}{|L(1, \chi)|^{2}} \\
& =\sum_{\substack{\chi \bmod q \\
\chi \neq \chi_{0}}}\left|\sum_{n \leq N} \chi(n)\right|^{2} \cdot\left|\sum_{1 \leq n \leq q^{4}} \frac{\chi(n) \mu(n)}{n}\right|^{2}+O\left(N^{2} q^{-1+\epsilon}\right) \\
& =\sum_{\chi \bmod q}\left|\sum_{n \leq N} \chi(n)\right|^{2} \cdot\left|\sum_{1 \leq n \leq q^{4}} \frac{\chi(n) \mu(n)}{n}\right|^{2}+O\left(N^{2} \ln ^{2} q\right) \\
& =\phi(q) \sum_{\substack{m \leq N \\
\text { m } \leq N N 1 \leq u \leq q^{4} 1 \leq v \leq q^{4} \\
m u \equiv n v(\bmod q)}}^{\prime} \frac{\mu(u) \mu(v)}{u v}+O\left(N^{2} \ln ^{2} q\right) \\
& =\phi(q) \sum_{m \leq N}^{\prime} \sum_{n \leq N 1 \leq u \leq q^{4}}^{\prime} \sum_{\substack{m u \leq n v \\
m}}^{\prime} \frac{\mu(u) \mu(v)}{u v}+O\left(N^{2} \ln ^{2} q\right)
\end{aligned}
$$




$$
\begin{aligned}
& +\phi(q) \sum_{m \leq N}^{\prime} \sum_{n \leq N}^{\prime} \sum_{1 \leq u \leq q^{4}}^{\prime} \sum_{1 \leq v \leq q^{4}}^{\prime} \frac{\mu(u) \mu(v)}{u v} \\
& \equiv M_{1}+M_{2}+M_{3} \text {, }
\end{aligned}
$$

where $\sum_{m \leq N}^{\prime}$ denotes the summation over all $m$ such that $1 \leq m \leq N$ and $(m, q)=1$.

Now we shall estimate $M_{1}$ and $M_{3}$ in (3.1) respectively. We have

$$
\begin{aligned}
& M_{3}=\phi(q) \sum_{\substack{m \leq N n \leq N 1 \leq u \leq q^{4} 1 \leq v \leq q^{4} \\
m u \equiv n v(\bmod q), m u \neq n v}}^{\prime} \frac{\mu(u) \mu(v)}{u v} \\
& =2 \phi(q) \sum_{m \leq N}^{\prime} \sum_{n \leq N}^{\prime} \sum_{1 \leq u \leq q^{4}}^{\prime} \sum_{1 \leq v \leq q^{4}}^{\prime} \frac{m \mu(u) \mu(v)}{m u v} \\
& \ll \phi(q) \sum_{m \leq N}^{\prime} \sum_{n \leq N}^{\prime} \sum_{1 \leq k \leq q^{4}} \sum_{1 \leq v \leq q^{4}}^{\prime} \frac{m}{(k q+n v) v} \\
& \ll N^{3} \ln ^{2} q .
\end{aligned}
$$

For $m u=n v$, let $(m, n)=d$, then $m=m_{1} d, n=n_{1} d$ and $\left(m_{1}, n_{1}\right)=1$. Then $m_{1} u=n_{1} v$. Let $v=m_{1} v_{1}$, then $u=n_{1} v_{1}$.

Let $A$ denotes the set of square-free numbers. Note that

$$
\begin{gathered}
\sum_{1 \leq n \leq N}^{\prime} 1=\frac{\phi(q)}{q} N+O\left(2^{\omega(q)}\right), \\
\sum_{\substack{h=1 \\
h \in A}}^{\infty} \frac{1}{h^{2}}=\sum_{n=1}^{\infty} \frac{\mu^{2}(n)}{n^{2}}=\frac{\zeta(2)}{\zeta(4)} \prod_{p \mid q}\left(1-\frac{1}{p^{2}+1}\right),
\end{gathered}
$$

where $\zeta(s)$ is the Riemann zeta-function.

We have

$$
\begin{aligned}
& M_{1}=\phi(q) \sum_{m \leq N} \sum_{n \leq N}^{\prime} \sum_{\substack{1 \leq u \leq q^{4} \\
m u=n v}}^{\prime} \sum_{1 \leq v \leq q^{4}}^{\prime} \frac{\mu(u) \mu(v)}{u v} \\
& =\phi(q) \sum_{d \leq N_{m_{1} \leq \frac{N}{d}}}^{\prime} \sum_{n_{1} \leq \frac{N}{d}}^{\prime} \sum_{\substack{1 \leq v_{1} \leq \min \left\{\frac{q^{4}}{m_{1}}, \frac{q^{4}}{n_{1}}\right\} \\
\prime}}^{\prime} \frac{\mu\left(n_{1} v_{1}\right) \mu\left(m_{1} v_{1}\right)}{m_{1} n_{1} v_{1}^{2}}
\end{aligned}
$$

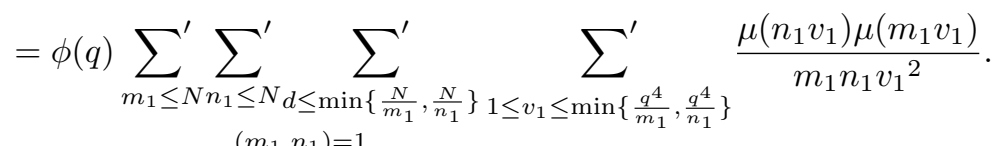

$$
\begin{aligned}
& \left(m_{1}, n_{1}\right)=1
\end{aligned}
$$


Obviously, $\mu\left(n_{1} v_{1}\right) \mu\left(m_{1} v_{1}\right)$ vanishes unless $n_{1}, m_{1}$ and $v_{1}$ are the squarefree numbers respectively and $\left(v_{1}, n_{1}\right)=\left(v_{1}, m_{1}\right)=1$ in which case it is equal to $\mu\left(n_{1}\right) \mu\left(m_{1}\right)$.

Then we have

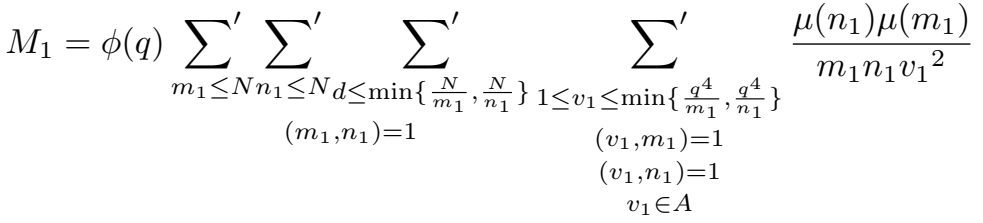

$$
\begin{aligned}
& =\phi(q) \sum_{d \leq N}^{\prime} \sum_{\substack{1 \leq v_{1} \leq q^{4} \\
v_{1} \in A}}^{\prime} \frac{1}{v_{1}^{2}}+2 \phi(q) \sum_{\substack{1 \leq n_{1}<N n_{1}<m_{1} \leq N \\
\left(m_{1}, n_{1}\right)=1}}^{\prime} \sum_{\substack { d \leq \frac{N}{m_{1}} \\
\begin{subarray}{c}{1 \leq v_{1} \leq \frac{q^{4}}{m_{1}} \\
\left(v_{1}, m_{1}\right)=1 \\
\left(v_{1}, n_{1}\right)=1 \\
v_{1} \in A{ d \leq \frac { N } { m _ { 1 } } \\
\begin{subarray} { c } { 1 \leq v _ { 1 } \leq \frac { q ^ { 4 } } { m _ { 1 } } \\
( v _ { 1 } , m _ { 1 } ) = 1 \\
( v _ { 1 } , n _ { 1 } ) = 1 \\
v _ { 1 } \in A } }\end{subarray}}^{\prime} \frac{\mu\left(n_{1}\right) \mu\left(m_{1}\right)}{m_{1} n_{1} v_{1}^{2}} \\
& =\frac{\phi^{2}(q)}{q} N \sum_{\substack{1 \leq v_{1} \leq q^{4} \\
v_{1} \in A}}^{\prime} \frac{1}{v_{1}^{2}}+O\left(\phi(q) 2^{\omega(q)}\right) \\
& +\frac{2 \phi^{2}(q)}{q} N \sum_{1 \leq n_{1}<N n_{1}<m_{1} \leq N}^{\prime} \frac{\mu\left(n_{1}\right) \mu\left(m_{1}\right)}{n_{1} m_{1}^{2}} \sum_{\substack{1 \leq v_{1} \leq q^{4} \\
\left(m_{1}, n_{1}\right)=1 \\
\left(v_{1}, m_{1}\right)=1 \\
\left(v_{1}, n_{1}\right)=1 \\
v_{1} \in A}}^{\prime} \frac{1}{v_{1}^{2}} \\
& =\frac{\zeta(2)}{\zeta(4)} \frac{\phi^{2}(q)}{q} N \prod_{p \mid q}\left(1-\frac{1}{p^{2}+1}\right)+O\left(\phi(q) 2^{\omega(q)}\right) \\
& +\frac{2 \phi^{2}(q)}{q} N \sum_{\substack{n_{1}=1 m_{1}=n_{1}+1 \\
\left(m_{1}, n_{1}\right)=1}}^{\infty} \frac{\mu\left(n_{1}\right) \mu\left(m_{1}\right)}{n_{1} m_{1}^{2}} \sum_{\substack{v_{1}=1 \\
\left(v_{1}, m_{1}\right)=1}}^{\infty} \frac{1}{v_{1}^{2}} \\
& \begin{array}{c}
\left(v_{1}, n_{1}\right)=1 \\
v_{1} \in A
\end{array} \\
& \equiv \frac{\zeta(2)}{\zeta(4)} \frac{\phi^{2}(q)}{q} N \prod_{p \mid q}\left(1-\frac{1}{p^{2}+1}\right)+O\left(\phi(q) 2^{\omega(q)}\right)+\Theta
\end{aligned}
$$

Using the properties of Möbius function we have

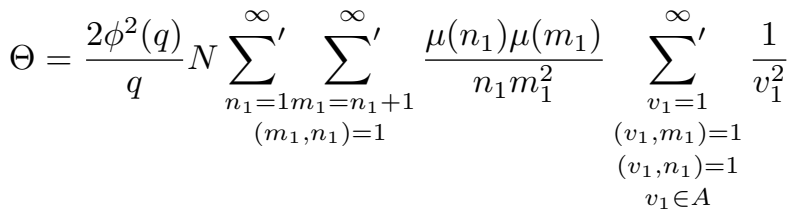




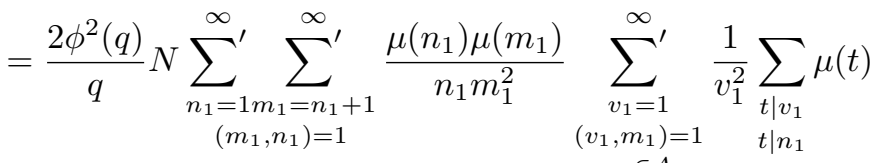

$$
\begin{aligned}
& v_{1} \in A \\
& =\frac{2 \phi^{2}(q)}{q} N \sum_{\substack{n_{1}=1 \\
\left(m_{1}, n_{1}\right)=1}}^{\infty} \sum_{\substack{m_{1}=n_{1}+1 \\
n_{1}}}^{\infty} \frac{\mu\left(n_{1}\right) \mu\left(m_{1}\right)}{n_{1} m_{1}^{2}} \sum_{\substack{t \mid n_{1} \\
\left(t, m_{1}\right)=1}}^{\prime} \frac{\mu(t)}{t^{2}} \sum_{\substack{k=1 \\
k \in A}}^{\infty} \frac{1}{k^{2}} \sum_{\substack{e|k \\
e| m_{1}}} \mu(e) \\
& =\frac{2 \phi^{2}(q)}{q} N \sum_{\substack{n_{1}=1 \\
\left(m_{1}, n_{1}\right)=1}}^{\infty} \sum_{\substack{m_{1}+1 \\
\left(t, m_{1}\right)=1}}^{\infty} \frac{\mu\left(n_{1}\right) \mu\left(m_{1}\right)}{n_{1} m_{1}^{2}} \sum_{\substack{t \mid n_{1} \\
(t)}}^{\prime} \frac{\mu(t)}{t^{2}} \sum_{e \mid m_{1}}^{\prime} \frac{\mu(e)}{e^{2}} \sum_{\substack{l=1 \\
l \in A}}^{\infty} \frac{1}{l^{2}} \\
& =\frac{2 \zeta(2)}{\zeta(4)} \frac{\phi^{2}(q)}{q} N \prod_{p \mid q}\left(1-\frac{1}{p^{2}+1}\right) \sum_{\substack{n_{1}=1 m_{1}=n_{1}+1 \\
\left(m_{1}, n_{1}\right)=1}}^{\infty} \frac{\mu\left(n_{1}\right) \mu\left(m_{1}\right)}{n_{1} m_{1}^{2}} \sum_{\substack{t \mid n_{1} \\
\left(t, m_{1}\right)=1}}^{\prime} \frac{\mu(t)}{t^{2}} \sum_{e \mid m_{1}}^{\prime} \frac{\mu(e)}{e^{2}} .
\end{aligned}
$$

So we can get

$$
\begin{aligned}
M_{1}= & \frac{\zeta(2)}{\zeta(4)} \frac{\phi^{2}(q)}{q} N \prod_{p \mid q}\left(1-\frac{1}{p^{2}+1}\right)+O\left(\phi(q) 2^{\omega(q)}\right) \\
& +\frac{2 \zeta(2)}{\zeta(4)} \frac{\phi^{2}(q)}{q} N \prod_{p \mid q}\left(1-\frac{1}{p^{2}+1}\right) \sum_{\substack{n_{1}=1 m_{1}=n_{1}+1 \\
\left(m_{1}, n_{1}\right)=1}}^{\infty} \frac{\mu\left(n_{1}\right) \mu\left(m_{1}\right)}{n_{1} m_{1}^{2}} \sum_{\substack{t \mid n_{1} \\
\left(t, m_{1}\right)=1}}^{\prime} \frac{\mu(t)}{t^{2}} \sum_{e \mid m_{1}}^{\prime} \frac{\mu(e)}{e^{2}} \\
= & \frac{\zeta(2)}{\zeta(4)} \frac{\phi^{2}(q)}{q} N \prod_{p \mid q}\left(1-\frac{1}{p^{2}+1}\right)(1+C)+O\left(\phi(q) 2^{\omega(q)}\right),
\end{aligned}
$$

where

$$
C=2 \sum_{\substack{n_{1}=1 \\\left(m_{1}, n_{1}\right)=1}}^{\infty} \sum_{\substack{m_{1}=n_{1}+1 \\\left(t, m_{1}\right)=1}}^{\infty} \sum_{\substack{t \mid n_{1} \\\left(t m_{1}\right.}}^{\prime} \frac{\mu\left(n_{1}\right) \mu\left(m_{1}\right) \mu(t) \mu(e)}{n_{1} m_{1}^{2} t^{2} e^{2}}
$$

is a constant depending only on $\bmod q$.

Combining (3.1) and above estimates we can get

$$
\begin{aligned}
& \sum_{\substack { \chi \bmod _{q} \neq \chi_{0} \\
\begin{subarray}{c}{\chi \\
\text { n }{ \chi \operatorname { m o d } _ { q } \neq \chi _ { 0 } \\
\begin{subarray} { c } { \chi \\
\text { n } } }\end{subarray}}\left|\sum_{n \leq N} \chi(n)\right|^{2} \cdot \frac{1}{|L(1, \chi)|^{2}} \\
= & \frac{\zeta(2)}{\zeta(4)} \frac{\phi^{2}(q)}{q} N \prod_{p \mid q}\left(1-\frac{1}{p^{2}+1}\right)(1+C)+O\left(\phi(q) 2^{\omega(q)}\right)+O\left(N^{3} \ln ^{2} q\right) .
\end{aligned}
$$

This proves Theorem 1. 
Now we prove Theorem 2. Let $q \geq 3$ be an integer, and $\chi$ be a Dirichlet character modulo $q$. From the properties of residue systems we have

$$
\begin{aligned}
|K(r, s, \chi ; q)|^{2} & =\sum_{\substack{a=1 \\
(a, q)=1}}^{q} \chi(a) e\left(\frac{r a+s \bar{a}}{q}\right) \sum_{\substack{b=1 \\
(b, q)=1}}^{q} \bar{\chi}(b) e\left(-\frac{r b+s \bar{b}}{q}\right) \\
& =\sum_{\substack{a=1 \\
(a, q)=1}}^{q} \sum_{\substack{b=1 \\
(b, q)=1}}^{q} \chi(a) e\left(\frac{r b(a-1)+s \bar{b}(\bar{a}-1)}{q}\right) \\
& =\phi(q)+\sum_{\substack{a=2 \\
(a, q)=1}}^{q} \sum_{\substack{b=1 \\
(b, q)=1}}^{q} \chi(a) e\left(\frac{r b(a-1)+s \bar{b}(\bar{a}-1)}{q}\right) .
\end{aligned}
$$

Note that

$$
(r, q)=(s, q)=(a, q)=1
$$

and

$$
(a-1, q)=(a \bar{a}-a, q)=(a(\bar{a}-1), q)=(\bar{a}-1, q)
$$

we have

$$
(r(a-1), s(\bar{a}-1), q)=(a-1, q) .
$$

According to the famous property of Kloosterman sums $K(r, s, q)$ (see [3]):

$$
|K(r, s, q)| \ll d(q) q^{1 / 2}(r, s, q)^{1 / 2},
$$

we can get

$$
\begin{aligned}
& \sum_{a=2}^{q} \sum_{\substack{b=1 \\
(b, q)=1}}^{q} \chi(a) e\left(\frac{r b(a-1)+s \bar{b}(\bar{a}-1)}{q}\right) \ll d(q) q^{\frac{1}{2}} \sum_{a=2}^{q}(a-1, q)^{\frac{1}{2}} \\
= & d(q) q^{\frac{1}{2}} \sum_{d \mid q} d^{\frac{1}{2}} \sum_{1 \leq l \leq q / d} 1 \ll q^{\frac{3}{2}} d^{2}(q) .
\end{aligned}
$$

It is clear that

$$
\begin{aligned}
& \sum_{\substack{\chi \bmod q \\
\chi \neq \chi_{0}}}\left|\sum_{n \leq N} \chi(n)\right|^{2} \cdot|K(r, s, \chi ; q)|^{2} \\
= & \phi(q) \sum_{\substack{\chi \bmod q \\
\chi \neq \chi_{0}}}\left|\sum_{n \leq N} \chi(n)\right|^{2}+\sum_{\substack{\chi \bmod q \\
\chi \neq \chi_{0}}}\left|\sum_{n \leq N} \chi(n)\right|^{2} \cdot \sum_{\substack{a=2 \\
(a, q)=1}}^{q} \sum_{\substack{b=1 \\
(b, q)=1}}^{q} \chi(a) e\left(\frac{r b(a-1)+s \bar{b}(\bar{a}-1)}{q}\right) \\
\equiv & E_{1}+E_{2} .
\end{aligned}
$$

Now we shall estimate $E_{1}$ and $E_{2}$ in (3.2) respectively. 
On one hand, from the orthogonality relationship for character sums we have

$$
\begin{aligned}
E_{1} & =\phi(q) \sum_{\substack{\chi \bmod q \\
\chi \neq \chi_{0}}}\left|\sum_{n \leq N} \chi(n)\right|^{2}=\phi(q) \sum_{m \leq N}^{\prime} \sum_{n \leq N}^{\prime} \sum_{\chi \bmod q} \chi(m \bar{n})+O\left(N^{2} \phi(q)\right) \\
& =\phi^{2}(q) \sum_{n \leq N}^{\prime} 1+O\left(N^{2} \phi(q)\right) \\
& =\frac{\phi^{3}(q)}{q} N+O\left(\phi^{2}(q) 2^{\omega(q)}\right)+O\left(N^{2} \phi(q)\right) .
\end{aligned}
$$

On the other hand, we may get

$$
\begin{aligned}
E_{2} & =\sum_{\substack{\chi \bmod q \\
\chi \neq \chi_{0}}}\left|\sum_{n \leq N} \chi(n)\right|^{2} \cdot \sum_{\substack{a=2 \\
(a, q)=1}}^{q} \sum_{\substack{b=1 \\
(b, q)=1}}^{q} \chi(a) e\left(\frac{r b(a-1)+s \bar{b}(\bar{a}-1)}{q}\right) \\
& =\phi(q) \sum_{n \leq N}^{\prime} \sum_{m \leq N}^{\prime} \sum_{\substack{a=2 \\
(a, q)=1 \\
a n \equiv m(\bmod q)}}^{q} \sum_{\substack{b=1 \\
(b, q)=1}}^{q} e\left(\frac{r b(a-1)+s \bar{b}(\bar{a}-1)}{q}\right)+O\left(N^{2} q^{\frac{3}{2}} d^{2}(q)\right) \\
& =\phi(q) \sum_{n \leq N}^{\prime} \sum_{m \neq N}^{\prime} \sum_{\substack{b=1 \\
m \neq n}}^{q} e\left(\frac{r b(m \bar{n}-1)+s \bar{b}(n \bar{m}-1)}{q}\right)+O\left(N^{2} q^{\frac{3}{2}} d^{2}(q)\right) \\
& =\Psi+O\left(N^{2} q^{\frac{3}{2}} d^{2}(q)\right) .
\end{aligned}
$$

Note that

$$
\begin{aligned}
\sum_{\substack{n \leq N \\
m \neq n}}^{\prime} \sum_{\substack{m \leq N \\
m \neq n}}^{\prime}(m-n, q)^{\frac{1}{2}} & =\sum_{\substack{d|q \\
d| m-n}} d^{\frac{1}{2}} \sum_{\substack{n \leq N \\
m \neq n}}^{\prime} \sum_{\substack{m \leq N \\
m \neq n}}^{\prime} 1 \\
& =\sum_{d \mid q} d^{\frac{1}{2}} \sum_{\substack{n \leq N \\
m \neq n, m \equiv n(\bmod d)}}^{\prime} \sum_{\substack{m \leq N \\
m, m}}^{\prime} 1 \leq \sum_{d \mid q} d^{\frac{1}{2}} \sum_{n \leq N} \frac{N-n}{d} \\
& \leq \sum_{d \mid q} d^{-\frac{1}{2}} \sum_{n \leq N} N \leq N^{2} d(q) .
\end{aligned}
$$

Then we have

$$
\Psi=\phi(q) \sum_{\substack{n \leq N \\ m \neq n}}^{\prime} \sum_{\substack{m \leq N \\ m \neq n}}^{\prime} \sum_{\substack{b=1 \\(b, q)=1}}^{q} e\left(\frac{r b(m \bar{n}-1)+s \bar{b}(n \bar{m}-1)}{q}\right)
$$




$$
\begin{aligned}
& \ll \phi(q) d(q) q^{\frac{1}{2}} \sum_{\substack{n \leq N \\
m \neq n}}^{\prime} \sum_{\substack{m \leq N \\
m \neq n}}^{\prime}(m \bar{n}-1, q)^{\frac{1}{2}} \\
& =\phi(q) d(q) q^{\frac{1}{2}} \sum_{\substack { n \leq N \\
\begin{subarray}{c}{m \neq n \\
m \leq N{ n \leq N \\
\begin{subarray} { c } { m \neq n \\
m \leq N } }\end{subarray}}^{\prime}(m-n, q)^{\frac{1}{2}} \\
& \ll N^{2} q^{\frac{1}{2}} d^{2}(q) \phi(q) .
\end{aligned}
$$

From (3.4) and (3.5) we know

$$
E_{2} \ll N^{2} q^{\frac{3}{2}} d^{2}(q)
$$

Using (3.2), (3.3), and (3.6) we can easily get

$$
\sum_{\substack{\chi \bmod q \\ \chi \neq \chi_{0}}}\left|\sum_{n \leq N} \chi(n)\right|^{2} \cdot|K(r, s, \chi ; q)|^{2}=\frac{\phi^{3}(q) N}{q}+O\left(N^{2} q^{\frac{3}{2}} d^{2}(q)\right) .
$$

This proves Theorem 2 .

Similarly, we also have

$$
\begin{aligned}
& \sum_{\substack{\chi \bmod q \\
\chi \neq \chi_{0}}}\left|\sum_{n \leq N} \chi(n)\right|^{4} \cdot|K(r, s, \chi ; q)|^{2} \\
= & \phi(q) \sum_{\substack{\chi \bmod q \\
\chi \neq \chi_{0}}}\left|\sum_{n \leq N} \chi(n)\right|^{4}+\sum_{\substack{\chi \bmod \\
\chi \neq \chi_{0}}}\left|\sum_{n \leq N} \chi(n)\right|^{4} \cdot \sum_{\substack{a=2 \\
(a, q)=1}}^{q} \sum_{\substack{b=1 \\
(b, q)=1}}^{q} \chi(a) e\left(\frac{r b(a-1)+s \bar{b}(\bar{a}-1)}{q}\right) \\
\equiv & A_{1}+A_{2} .
\end{aligned}
$$

Now we shall estimate $A_{1}$ and $A_{2}$ in (3.7) respectively. Applying orthogonality relationship for character sums and Lemma 2 we have

$$
\begin{aligned}
& A_{1}=\phi(q) \sum_{m \leq N}^{\prime} \sum_{n \leq N}^{\prime} \sum_{u \leq N}^{\prime} \sum_{v \leq N}^{\prime} \sum_{\chi \bmod q} \chi(m u \bar{n} \bar{v})+O\left(N^{4} \phi(q)\right) \\
& =\phi^{2}(q) \sum_{m \leq N}^{\prime} \sum_{\substack{n \leq N \\
m u=n v}}^{\prime} \sum_{\substack{u \leq N \\
v \leq N}}^{\prime} 1+O\left(N^{4} \phi(q)\right) \\
& =\phi^{2}(q) \sum_{m \leq N}^{\prime} \sum_{n \leq N}^{\prime} \sum_{d \leq \min \left\{\frac{N}{m}, \frac{N}{n}\right\}}^{\prime} \sum_{\substack{v \leq \min \left\{\frac{N}{m}, \frac{N}{n}\right\} \\
(m, n)=1}}^{\prime} 1+O\left(N^{4} \phi(q)\right)
\end{aligned}
$$




$$
\begin{aligned}
& =\phi^{2}(q)\left(\sum_{m \leq N}^{\prime} 1\right)^{2}+2 \phi^{2}(q) \sum_{\substack{1<m \leq N \\
(m, n)=1 \\
(m \leq n<m}}^{\prime} \sum_{\substack{v \leq \frac{N}{m} \\
(}}^{\prime}\left(\sum^{\prime}\right)^{2}+O\left(N^{4} \phi(q)\right) \\
& =\frac{\phi^{4}(q) N^{2}}{q^{2}}+\frac{2 \phi^{4}(q) N^{2}}{q^{2}} \sum_{\substack{1<m \leq N \leq n \leq n \\
(m, n)=1}}^{\prime} \sum_{\substack{1 \leq n<m \\
m^{2}}}^{\prime} \frac{1}{m^{2}}+O\left(\phi^{3}(q) q^{-1} N 2^{\omega(q)}\right)+O\left(N^{4} \phi(q)\right) \\
& =\frac{12 \phi^{6}(q) N^{2}}{\pi^{2} q^{4}} \prod_{p \mid q}\left(1-\frac{1}{p^{2}}\right)^{-1} \ln N+O\left(\phi^{4}(q) q^{-2} N^{2} d(q)\right) .
\end{aligned}
$$

We also have

$$
\begin{aligned}
A_{2}= & \sum_{\substack{\chi \bmod q \\
\chi \neq \chi_{0}}}\left|\sum_{n \leq N} \chi(n)\right|^{4} \cdot \sum_{\substack{a=2 \\
(a, q)=1}}^{q} \sum_{\substack{b=1 \\
(b, q)=1}}^{q} \chi(a) e\left(\frac{r b(a-1)+s \bar{b}(\bar{a}-1)}{q}\right) \\
= & \phi(q) \sum_{n_{1} \leq N}^{\prime} \sum_{n_{2} \leq N}^{\prime} \sum_{m_{1} \leq N}^{\prime} \sum_{m_{2} \leq N}^{\prime} \sum_{\substack{a=2 \\
(a, q)=1}}^{q} \sum_{\substack{b=1 \\
(b, q)=1}}^{q} e\left(\frac{r b(a-1)+s \bar{b}(\bar{a}-1)}{q}\right)+O\left(N^{4} q^{\frac{3}{2}} d^{2}(q)\right) \\
= & \phi(q) \sum_{n_{1} \leq N}^{\prime} \sum_{n_{2} \leq N}^{\prime} \sum_{n_{1} m_{1} \leq N}^{\prime} \sum_{m_{2} \leq N}^{\prime} \sum_{\substack{b=1 \\
(b, q)=1}}^{q} e\left(\frac{r b\left(n_{2} m_{2} \overline{n_{1}} \bar{m}_{1}-1\right)+s \bar{b}\left(n_{1} m_{1} \overline{n_{2}} \bar{m}_{2}-1\right)}{q}\right) \\
& +O\left(N^{4} q^{\frac{3}{2}} d^{2}(q)\right) \\
= & \Omega+O\left(N^{4} q^{\frac{3}{2}} d^{2}(q)\right) .
\end{aligned}
$$

Note that

$$
\begin{aligned}
& \sum_{n_{1} \leq N}^{\prime} \sum_{\substack{n_{2} \leq N \\
n_{1} m_{1} \neq n_{2} \leq m_{2}}}^{\prime} \sum_{m_{1} \leq N}^{\prime} \sum_{m_{2} \leq N}^{\prime}\left(n_{2} m_{2}-n_{1} m_{1}, q\right)^{\frac{1}{2}} \\
& =\sum_{\substack{d|q \\
d| n_{2} m_{2}-n_{1} m_{1}}} d^{\frac{1}{2}} \sum_{n_{1} \leq N}^{\prime} \sum_{\substack{n_{2} \leq N \\
n_{1} m_{1} \neq n_{2} m_{2}}}^{\prime} \sum_{m_{1} \leq N}^{\prime} \sum_{m_{2} \leq N}^{\prime} 1 \\
& =\sum_{\substack{d \mid q \\
n_{2} m_{2} \equiv n_{1} m_{1}(\bmod d)}} d^{\frac{1}{2}} \sum_{n_{1} \leq N}^{\prime} \sum_{\substack{n_{2} \leq N \\
n_{1} m_{1} \neq n_{2} m_{2}}}^{\prime} \sum_{\substack{m_{1} \leq N \\
m_{2} \leq N}}^{\prime} \sum^{\prime} 1 \\
& \leq \sum_{d \mid q} d^{\frac{1}{2}} \sum_{n_{1} \leq N}^{\prime} \sum_{n_{2} \leq N}^{\prime} \sum_{m_{1} \leq N}^{\prime} \frac{N}{d} \leq N^{4} d(q) \text {. }
\end{aligned}
$$


Then we may get

$$
\begin{aligned}
& \Omega=\phi(q) \sum_{n_{1} \leq N}^{\prime} \sum_{n_{2} \leq N}^{\prime} \sum_{\substack{m_{1} \leq N \\
n_{1} m_{1} \neq n_{2} m_{2}}}^{\prime} \sum_{m_{2} \leq N}^{\prime} \sum_{\substack{b=1 \\
(b, q)=1}}^{q} e\left(\frac{r b\left(n_{2} m_{2} \bar{n}_{1} \bar{m}_{1}-1\right)+s \bar{b}\left(n_{1} m_{1} \overline{n_{2}} \bar{m}_{2}-1\right)}{q}\right) \\
& \ll \phi(q) d(q) q^{\frac{1}{2}} \sum_{n_{1} \leq N}^{\prime} \sum_{n_{2} \leq N}^{\prime} \sum_{m_{1} \leq N}^{\prime} \sum_{m_{2} \leq N}^{\prime}\left(n_{2} m_{2} \overline{n_{1}} \bar{m}_{1}-1, q\right)^{\frac{1}{2}} \\
& =\phi(q) d(q) q^{\frac{1}{2}} \sum_{n_{1} \leq N}^{\prime} \sum_{\substack{n_{2} \leq N \\
n_{1} m_{1} \neq n_{2} m_{2}}}^{\prime} \sum_{m_{1} \leq N}^{\prime} \sum_{m_{2} \leq N}^{\prime}\left(n_{2} m_{2}-n_{1} m_{1}, q\right)^{\frac{1}{2}} \\
& \ll N^{4} q^{\frac{1}{2}} d^{2}(q) \phi(q) .
\end{aligned}
$$

From (3.9) and (3.10) we know

$$
A_{2} \ll N^{4} q^{\frac{3}{2}} d^{2}(q) .
$$

Therefore, combining (3.7), (3.8), and (3.11) we can immediately get

$$
\begin{aligned}
& \sum_{\substack{\chi \bmod q \\
\chi \neq \chi_{0}}}\left|\sum_{n \leq N} \chi(n)\right|^{4} \cdot|K(r, s, \chi ; q)|^{2} \\
= & \frac{12 \phi^{6}(q) N^{2}}{\pi^{2} q^{4}} \prod_{p \mid q}\left(1-\frac{1}{p^{2}}\right)^{-1} \ln N+O\left(\phi^{4}(q) q^{-2} N^{2} d(q)\right) .
\end{aligned}
$$

This completes the proof of Theorem 3 .

Theorem 4 follows from Theorem 3 and Lemma 3.

Acknowledgements. The authors would like to express their sincere gratitude to the referee for his/her very valuable suggestions and detailed comments on improving this paper. The authors wish to thank Professor W. Zhang for his encouragements.

\section{References}

[1] T. M. Apostol, Introduction to Analytic Number Theory, Undergraduate Texts in Mathematics. Springer-Verlag, New York-Heidelberg, 1976.

[2] S. Chowla, On Kloosterman's sum, Norkse Vid. Selbsk. Fak. Frondheim 40 (1967), 70-72.

[3] T. Estermann, On Kloosterman's sum, Mathematica 8 (1961), 83-86.

[4] H. L. Montgomery and R. C. Vaughan, Mean values of character sums, Canad. J. Math. 31 (1979), no. 3, 476-487.

[5] C. D. Pan and C. B. Pan, Goldbach Conjecture, Science Press, Beijing, 1981.

[6] Z. Xu and W. Zhang, On the $2 k$-th power mean of the character sums over short intervals, Acta Arith. 121 (2006), no. 2, 149-160.

[7] W. Zhang, On the mean value of L-functions with the weight of character sums, J. Number Theory 128 (2008), no. 8, 2459-2466. 
[8] _ On the general Kloosterman sums and its fourth power mean, J. Number Theory 104 (2004), no. 1, 156-161.

[9] W. Zhang, Y. Yi, and X. He, On the $2 k$-th power mean of Dirichlet L-functions with the weight of general Kloosterman sums, J. Number Theory 84 (2000), no. 2, 199-213.

XiAOBENG ZHANG

Department of Mathematics

NoRThWEST UNIVERSITY

Xi'an, ShaAnxi, P. R. China

AND

Department of Applied Mathematics and Physics

Xi'An University of Post and Telecommunications

Xi'an, ShaAnxi, P. R. China

E-mail address: bengbeng1231@yahoo.com.cn

HuAning LiU

Department of Mathematics

Northwest University

Xi'an, ShaAnxi, P. R. China

E-mail address: hnliumath@hotmail.com 